\title{
Variability of extracorporeal cardiopulmonary resuscitation utilization for refractory adult out-of- hospital cardiac arrest: an international survey study
}

\author{
Patrick J. Coppler ${ }^{1}$, Benjamin S. Abella ${ }^{2}$, Clifton W. Callaway ${ }^{1}$, \\ Minjung Kathy Chae ${ }^{3}$, Seung Pill Choi ${ }^{4}$, Jonathan Elmer ${ }^{1,5}$, \\ Won Young Kim ${ }^{6}$, Young-Min Kim${ }^{4}$, Michael Kurz ${ }^{7}$, Joo Suk $\mathrm{Oh}^{8}$, \\ Joshua C. Reynolds ${ }^{9}$, Jon C. Rittenberger ${ }^{1}$, Kelly N. Sawyer ${ }^{1}$,
} Chun Song Youn ${ }^{4}$, Byung Kook Lee ${ }^{10}$, David F. Gaieski ${ }^{11}$, on behalf of the Korean Hypothermia Network Investigators and the National Post-Arrest Research Consortium*

\begin{abstract}
'Department of Emergency Medicine, University of Pittsburgh School of Medicine, Pittsburgh, PA, USA ${ }^{2}$ Department of Emergency Medicine, Perelman School of Medicine at the University of Pennsylvania, Philadelphia, PA, USA

${ }^{3}$ Department of Emergency Medicine, Ajou University Hospital, Suwon, Korea

${ }^{4}$ Department of Emergency Medicine, The Catholic University of Korea College of Medicine, Seoul, Korea ${ }^{5}$ Department of Critical Care Medicine, University of Pittsburgh School of Medicine, Pittsburgh, PA, USA ${ }^{6}$ Department of Emergency Medicine, Asan Medical Center, Ulsan University College of Medicine, Seoul, Korea ${ }^{7}$ Department of Emergency Medicine, University of Alabama School of Medicine, Birmingham, AL, USA ${ }^{8}$ Department of Emergency Medicine, Uijeongbu St. Mary's Hospital, The Catholic University of Korea College of Medicine, Uijeongbu, Korea

${ }^{9}$ Department of Emergency Medicine, Michigan State University College of Human Medicine, Grand Rapids, MI, USA

${ }^{10}$ Department of Emergency Medicine, Chonnam National University Hospital, Gwangju, Korea

${ }^{11}$ Department of Emergency Medicine, Sidney Kimmel Medical College at Thomas Jefferson University Hospital, Philadelphia, PA, USA
\end{abstract}

Objective A growing interest in extracorporeal cardiopulmonary resuscitation (ECPR) as a rescue strategy for refractory adult out-of-hospital cardiac arrest (OHCA) currently exists. This study aims to determine current standards of care and practice variation for ECPR patients in the USA and Korea.

Methods In December 2015, we surveyed centers from the Korean Hypothermia Network (KORHN) Investigators and the US National Post-Arrest Research Consortium (NPARC) on current targeted temperature management and ECPR practices. This project analyzes the subsection of questions addressing ECPR practices. We summarized survey results using descriptive statistics.

Results Overall, 9 KORHN and 4 NPARC centers reported having ECPR programs and had complete survey data available. Two KORHN centers utilized extracorporeal membrane oxygenation only for postarrest circulatory support in patients with refractory shock and were excluded from further analysis. Centers with available ECPR generally saw a high volume of OHCA patients (10/11 centers care for $>75$ OHCA a year). Location of, and providers trained for cannulation varied across centers. All centers in both countries (KORHN 7/7, NPARC 4/4) treated comatose ECPR
elSSN: 2383-4625

Received: 11 March 2017

Revised: 2 May 2017

Accepted: 8 May 2017

Correspondence to: Byung Kook Lee Department of Emergency Medicine, Chonnam National University Hospital, 42 Jebong-ro, Dong-gu, Gwangju 61469, Korea

E-mail: bbukkuk@hanmail.net

*A full list of investigators of the Korean Hypothermia Network Investigators and the National PostArrest Research Consortium are listed at the end of the article.

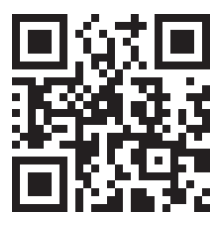

How to cite this article:

Coppler PJ, Abella BS, Callaway CW, Chae MK, Choi SP, Elmer J, Kim WY, Kim YM, Kurz M, Oh JS, Reynolds JC, Rittenberger JC, Sawyer KN, Youn CS, Lee BK, Gaieski DF; Korean Hypothermia Network Investigators and the National Post-Arrest Research Consortium. Variability of extracorporeal cardiopulmonary resuscitation utilization for refractory adult out-of-hospital cardiac arrest: an international survey study. Clin Exp Emerg Med 2018;5(2):100-106.

This is an Open Access article distributed under the terms of the Creative Commons Attribution Non-Commercial License (http:// creativecommons.org/licenses/by-nc/4.0/). 
patients with targeted temperature management. All NPARC centers and four of seven KORHN centers reported having a standardized hospital protocol for ECPR. Upper age cutoff for eligibility ranged from 60 to 75 years. No absolute contraindications were unanimous among centers.

Conclusion A wide variability in practice patterns exist between centers performing ECPR for refractory OHCA in the US and Korea. Standardized protocols and shared research databases might inform best practices, improve outcomes, and provide a foundation for prospective studies.

Keywords Extracorporeal membrane oxygenation; Heart arrest; Cardiopulmonary resuscitation

Capsule
Wummat is already known
Extracorporeal cardiopulmonary resuscitation is becoming an increasingly available modality for the treatment of re-
fractory cardiac arrest.
What is new in the current study
We report variability in extracorporeal cardiopulmonary resuscitation patient selection and practice characteristics.
Future guidelines and statements of best practices are needed. Comparison of data across centers may be limited by
this practice variability.

\section{INTRODUCTION}

Out-of-hospital cardiac arrest (OHCA) is a significant global health burden with an annual incidence ranging from 29 to 55 cases per 100,000 population, of which only $2 \%$ to $11 \%$ survive to hospital discharge. ${ }^{1}$ Approximately $75 \%$ of adult patients treated for OHCA do not achieve return of spontaneous circulation (ROSC) despite conventional cardiopulmonary resuscitation (CPR) and advanced cardiac life support. ${ }^{2}$ Many patients who do not survive to hospital admission after OHCA have autopsy evidence of acute coronary occlusion. ${ }^{3}$ Similarly, patients with other reversible etiologies of cardiac arrest, such as fulminant myocarditis, variant angina, and massive pulmonary embolism, are sometimes refractory to standard resuscitation measures. ${ }^{4-6}$

Emergent extracorporeal cardiopulmonary resuscitation (ECPR), which consists of implementing veno-arterial extracorporeal membrane oxygenation (VA ECMO), is a potential therapy for patients with a reversible cause of arrest who are refractory to conventional CPR and advanced cardiac life support interventions. Historically, ECPR was used primarily to treat pediatric in hospital cardiac arrest patients..$^{7-9}$ Over time, the scope of ECPR has expanded to adult in hospital cardiac arrest, and now to select candidates with OHCA. A 2010 Japanese review of multiple case series, originally published in Japanese language journals, demonstrated a $26.7 \%$ survival rate for more than 500 OHCA patients treated with ECPR. ${ }^{10}$ In 2016, a meta-analysis of observational evidence suggested improved 1-year survival (relative risk, 1.96; 95\% confidence interval, 1.00 to 3.87 ) and 1-year neurological outcome defined as a cerebral performance category of one or two (relative risk, 2.63; 95\% confidence interval, 1.11 to 6.21 ). ${ }^{11}$ As the necessary equipment becomes smaller and more portable, ECPR has also been successfully deployed in both the emergency department (ED) and in prehospital settings. ${ }^{12-16}$ The 2015 American Heart Association guidelines state, "In settings where it can be rapidly implemented, ECPR may be considered for select cardiac arrest patients for whom the suspected etiology of the cardiac arrest is potentially reversible during a limited period of mechanical cardiorespiratory support (class IIb, LOE C-LD)." ${ }^{17}$

Though a growing interest in ECPR exists, relatively little is known about the current practice patterns, including patient selection criteria, programmatic structure, and cannulation practices. One recent review of the published literature found a wide variation in indications such as CPR duration, age, and initial rhythm when considering ECPR as well as use of postarrest therapies such as coronary angiography and therapeutic hypothermia. ${ }^{18}$ In addition, the Extracorporeal Resuscitation Consortium (ERECT) Research Group recently published a survey data from 99 Emergency Life Support Organization (ELSO) members who report performing ECPR and found that 50\% of the respondents had performed ECPR in the ED, but more than $90 \%$ of cannulations were performed by consulting cardiothoracic (CT) surgeons. ${ }^{19}$ Further information is needed. In addition to the published literature, we 
surveyed ECPR practice in centers participating in one of two large national research consortia in the US and Korea. We hypothesized that the indications for ECPR, cannulation practices, and use of targeted temperature management (TTM) would vary among centers and countries.

\section{METHODS}

We performed an internet-based survey (Qualtrics, Provo, UT, USA) of postarrest care practices including $\Pi M$, neurological prognostication, and ECPR in English and Korean, which we have previously described in detail. ${ }^{20}$ A dedicated 18-question sub-survey focused specifically on ECPR. The survey was distributed to 35 sites across Korea (Korean Hypothermia Network, KORHN) and 7 sites across the US (National Post-Arrest Research Consortium, NPARC). ${ }^{21-23}$ The investigators from these sites were also asked to submit any hardcopy of institutional ECPR protocols. The University of Pittsburgh institutional review board granted institutional review board exemption for all aspects of this study.

We asked a single investigator from each KORHN and NPARC site to complete the survey. Only centers with an ECPR program completed the ECPR subsection. Investigators were instructed to answer survey questions based on local ECPR institutional protocols and practice for adult OHCA patients. The survey was sent in December 2015 and final data collection was completed in January 2016. Survey responses were included in the analysis if $>50 \%$ of questions were answered. We performed a descriptive statistics using the Stata ver. 13.1 (StataCorp., College Station, TX, USA).

\section{RESULTS}

Of the 33 KORHN and 7 NPARC surveys completed, 100\% of the institutions completed the main survey. Overall, 9 KORHN and 4 NPARC institutions reported having an ECPR program and had complete survey data available. Centers using ECPR generally saw a high volume of OHCA, with 12/13 centers caring for $>75$ patients per year (Table 1). Survey responders were predominantly Emergency Medicine specialists (KORHN 9/9, NPARC 3/4). In two KORHN centers, ECPR was utilized only for postarrest circulatory support in patients with refractory shock and these were excluded from further analysis. The ECPR annual caseload ranged from 0 to 30 with a median of 5 cases treated per year (interquartile range [IOR] 3 to 10) (KORHN median 5, IQR 2 to 10; NPARC median 6, IQR 5 to 19) (Fig. 1).

Location of cannulation and provider trained with cannulation for ECPR varied among centers (Table 1). Both KORHN and NPARC centers cannulate in the ED (KORHN 3/7, NPARC 3/4); however,
Table 1. ECPR cannulation characteristics

\begin{tabular}{|c|c|c|}
\hline Characteristics & NPARC & KORHN \\
\hline Total annual out-of-hospital cardiac arrest volume & $n=4$ & $n=7$ \\
\hline$<50$ & 0 & 0 \\
\hline $50-74$ & 0 & 1 \\
\hline $75-99$ & 1 & 1 \\
\hline $100-124$ & 2 & 1 \\
\hline$>125$ & 1 & 4 \\
\hline \multicolumn{3}{|l|}{ Organized ECPR team } \\
\hline Yes & 3 & 3 \\
\hline No & 1 & 4 \\
\hline \multicolumn{3}{|l|}{ 24-hr ECPR capable } \\
\hline Yes & 2 & 5 \\
\hline No & 2 & 2 \\
\hline \multicolumn{3}{|l|}{ Preferred location } \\
\hline Emergency department & 3 & 3 \\
\hline Operating room & 0 & 0 \\
\hline Cardiac catheterization lab & 0 & 2 \\
\hline Procedure suite & 0 & 0 \\
\hline Depends on scenario ${ }^{\text {a) }}$ & 1 & 2 \\
\hline \multicolumn{3}{|l|}{ Cannulating specialty } \\
\hline Emergency medicine physician & 0 & 4 \\
\hline Cardiothoracic surgeon & 4 & 5 \\
\hline Surgical intensivist & 1 & 0 \\
\hline Interventional cardiologist & 0 & 4 \\
\hline \multicolumn{3}{|l|}{ Cannulation final decision } \\
\hline Attending of record & 0 & 1 \\
\hline Physician in charge of cannulation service & 2 & 5 \\
\hline Other ${ }^{b)}$ & 2 & 1 \\
\hline Time from vessel puncture to ECMO flow (min) & $n=1$ & $n=7$ \\
\hline 15 & 1 & 2 \\
\hline 20 & 0 & 2 \\
\hline 25 & 0 & 1 \\
\hline 30 & 0 & 2 \\
\hline
\end{tabular}

ECPR, extracorporeal cardiopulmonary resuscitation; NPARC, National Post-Arrest Research Consortium; KORHN, Korean Hypothermia Network; ECMO, extracorporeal membrane oxygenation.

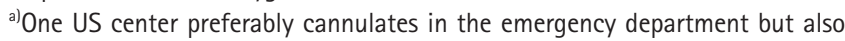
uses cardiac catheterization lab or operating room. Two of two Korean centers preferably cannulate in emergency department but also use cardiac catheterization lab. ${ }^{b}$ US: 1 team decision, 1 postarrest service + cardiothoracic surgeon. Korea: either emergency department or cardiothoracic surgery attending.

other centers also primarily cannulate in the ED but in various locations depending on the situational factors (KORHN 4/7, NPARC 1/4). Patients were primarily cannulated by emergency physicians (4/7), CT surgeons (5/7), and interventional cardiologists (4/7) in KORHN centers, whereas CT surgeons (4/4) cannulated in all NPARC centers. All centers in both countries (KORHN 7/7, NPARC 4/4) used TTM for patients with persistent coma after initiating ECPR and ROSC.

All NPARC centers and four of seven KORHN centers reported 


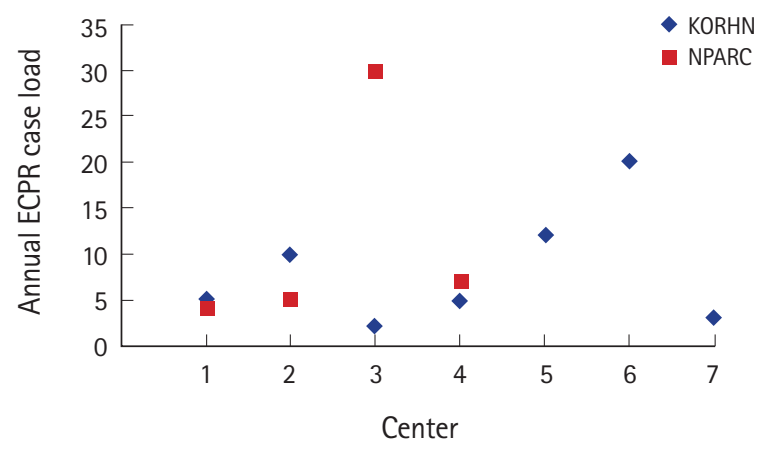

Fig. 1. Annual extracorporeal cardiopulmonary resuscitation (ECPR) case load per center. KORHN, Korean Hypothermia Network; NPARC, National Post-Arrest Research Consortium.

having a standardized hospital protocol for ECPR (Table 2). Upper age cutoff for eligibility ranged from 60 to 75 years. No absolute contraindications were unanimous among participating centers. A number of both NPARC and KORHN centers reported cannulating a high proportion of patients that meet exclusion criteria for ECPR.

\section{DISCUSSION}

We found a high level of variation in ECPR practices among the surveyed large volume cardiac arrest centers in South Korea and the US. Moreover, variability exists between centers in each country. These differences are an important potential source of bias or confounding in studies evaluating the effectiveness of ECPR in a refractory cardiac arrest.

Practice variability existed between NPARC and KORHN institutions. Organized protocols were more common at NPARC than KORHN institutions. KORHN and NPARC investigators report different locations and specialists responsible for cannulation and initiation of VA ECMO. In KORHN centers, cannulation is frequently performed in the cardiac catheterization laboratory, but not in that of NPARC centers. Institutions from both countries cannulate in the ED. In KORHN centers, multiple specialists are responsible for cannulation, while only surgical intensivists or cardiothoracic surgeons currently cannulate in NPARC centers. Other surveys have found that CT surgeons cannulate in a majority of US ECPR centers. ${ }^{19}$ However, no interventional cardiologists were found to be primary cannulators in the surveyed NPARC centers. Absence of these specialists upon arrival of the ECPR patient in the hospital may result in delays to functional VA ECMO or no cannulation attempt at all. This may be one of the reasons for the low rate of cannulating patients who meet the exclusion criteria found in NPARC centers when compared to KORHN centers. Sim-
Table 2. ECPR selection criteria

\begin{tabular}{|c|c|c|}
\hline Details on criteria & NPARC & KORHN \\
\hline Standardized institutional ECPR protocol & $n=4$ & $n=7$ \\
\hline Yes & 4 & 5 \\
\hline No & 0 & 2 \\
\hline Inclusion criteria & $n=4$ & $n=4$ \\
\hline Age $<75 \mathrm{yr}$ & 0 & 2 \\
\hline Age $<70 \mathrm{yr}$ & 1 & 1 \\
\hline Age $<65 \mathrm{yr}$ & 1 & 0 \\
\hline Age $<60 \mathrm{yr}$ & 1 & 1 \\
\hline Bystander CPR & 1 & 3 \\
\hline Shockable initial rhythm & 1 & 1 \\
\hline Sustained VF cardiac arrest despite 30 min of resuscitation & 1 & 3 \\
\hline Exclusion criteria & $n=4$ & $n=4$ \\
\hline Collapse to CPR time $>30 \mathrm{~min}$ & 0 & 1 \\
\hline Unwitnessed event & 3 & 3 \\
\hline Major preexisting medical conditions ${ }^{\text {a) }}$ & 3 & 3 \\
\hline Known aortic dissection & 2 & 3 \\
\hline Aortic insufficiency & 1 & 0 \\
\hline Recent CVA & 0 & 2 \\
\hline Traumatic arrest & 2 & 3 \\
\hline Body habitus & 3 & 2 \\
\hline Obvious vascular disease & 2 & 1 \\
\hline Preexisting neurological disease & 3 & 3 \\
\hline Poor baseline neuro or functional status & 2 & 3 \\
\hline Initial asystolic rhythm & 2 & 2 \\
\hline Initial PEA rhythm & 2 & 0 \\
\hline Other & 0 & 0 \\
\hline Patients cannulated who met exclusion & $n=4$ & $n=3$ \\
\hline Never & 0 & 0 \\
\hline $1 \%-10 \%$ & 3 & 1 \\
\hline $11 \%-20 \%$ & 1 & 0 \\
\hline $21 \%-30 \%$ & 0 & 0 \\
\hline$>30 \%$ & 0 & 2 \\
\hline
\end{tabular}

ECPR, extracorporeal cardiopulmonary resuscitation; NPARC, National Post-Arrest Research Consortium; KORHN, Korean Hypothermia Network; CPR, cardiopulmonary resuscitation; $V F$, ventricular fibrillation; $C V A$, cerebral vascular accident; PEA, pulseless electrical activity.

${ }^{a}$ Debilitating medical conditions associated with minimal odds of good outcome.

ilarly, patients who meet the inclusion criteria for cannulation may be missed in this model. Future work should determine the percentage of patients who meet the criteria for cannulation but are not treated using this therapy.

Some NPARC protocols exclude pulseless electrical activity arrest, prolonged ventricular fibrillation arrest, and age $>70$ years, whereas KORHN sites do not exclude these criteria. NPARC sites appear to be more conservative in providing ECPR when compared to KORHN sites, because they may have experienced few ECPR cases, the presence of cultural differences in resuscitation care, or centers averse to cannulating in heroic situations. ${ }^{20}$

These findings highlight the need for future research of best 
practices as well as the development of formalized hospital, regional, and national guidelines for ECPR. Variations in criteria for patient selection, eligibility, and contraindications each introduce potential for confounding in clinical research. These factors must be controlled in both observational and experimental study designs. Similar to the Utstein style standard variables collected in cardiac arrest registries, ${ }^{24}$ a consensus set of core variables should be developed for ECPR registries to facilitate pooled analyses.

Practice variability is likely unavoidable and will require tailoring of the idiosyncrasies of each hospital/healthcare system. Both selection criteria and the nuances in cannulation logistics (location, specialty service, etc.) may require some inherent unavoidable practice variability. Ortega-Deballon et al. ${ }^{18}$ conducted a systematic review of available literature concerning ECPR studies and found significant variability in selection criteria including patient age, initial rhythm, and duration of no flow and low flow times. The ERECT research group surveyed centers that had submitted ECPR cases to the ELSO. Most of the 70 included centers did not have formal selection criteria for ECPR.

Our study has several limitations. The large volume centers in the US and Korea that participated in this study may not reflect the full extent of ECPR practice variability. However, these large centers represent the majority of facilities that can provide emergent ECPR. Other investigators have found similar variability in ECPR practices. ${ }^{18}$ Additional variability may exist outside the context of the provided survey questions. Finally, we did not survey the centers with regard to sedation, neuromuscular blockade, or withdrawal of care specifically for ECPR patients.

A wide variability exists at institutions performing ECPR for refractory cardiac arrest in Korea and the US, and we reported national and international variability. This variability may confound both observational and experimental research on ECPR. It also underscores the need for standardized guidelines and definitions of common ECPR variables to facilitate best practices, shared data, and research collaboration.

\section{CONFLICT OF INTEREST}

No potential conflict of interest relevant to this article was reported.

\section{ACKNOWLEDGMENTS}

Jonathan Elmer's research time is supported by National Institutes of Health grant (5K12 HL109068).

\section{Korean Hypothermia Network investigators}

Ji Hoon Kim, Bucheon St. Mary's Hospital, The Catholic University of Korea; Kyu Nam Park, Seoul St. Mary's Hospital, The Catholic University of Korea; Won Jung Jeong, St. Vincent's Hospital, The Catholic University of Korea; Seung Pill Choi, Yeouido St. Mary's Hospital, The Catholic University of Korea; Mi Jin Lee, Kyungpook National University School of Medicine; Jong-Seok Lee, Kyung Hee University Medical Center; Su Jin Kim, Korea University College of Medicine; Tae Chang Jang, Catholic University of Daegu College of Medicine; Inbyung Kim, Myongji Hospital, Seonam University College of Medicine; Yong Hwan Kim, Samsung Changwon Hospital, Sungkyunkwan University School of Medicine; Won Young Kim, Asan Medical Center, University of Ulsan College of Medicine; Jonghwan Shin, Seoul National University Boramae Medical Center; Ji Hwan Lee, Yonsei University College of Medicine; Hyung Jun Moon, Soonchunhyang University Hospital; Giwoon Kim, Soonchunhyang University Hospital; Wook-jin Choi, Ulsan University Hospital, University of Ulsan College of Medicine; Joo Suk Oh, Uijeongbu St. Mary's Hospital, The Catholic University of Korea; Chul Han, Ewha Womans University Mokdong Hospital; Byung Kook Lee, Chonnam National University Medical School; Taeoh Jeong, Research Institute of Clinical Medicine of Chonbuk National University and Chonbuk National University Hospital; Dong Hoon Lee, Chung-Ang University; Min Jin Hong, Chungbuk National University Hospital; Gyu Chong Cho, Hallym University; Young Hwan Lee, Hallym University Sacred Heart Hospital, Hallym University Medical Center; Youdong Sohn, Hallym University Sacred Heart, Hospital, Hallym University Medical Center; In Soo Cho, Hanil General Hospital; Je Sung You, Yonsei University College of Medicine; Changsun Kim, Hanyang University Guri Hospital; Kyoung-Chul Cha, Yonsei University Wonju College of Medicine; Soo Hyung Cho, Chosun University Hospital.

\section{National Postarrest Research Consortium investigators}

Michael Donnino, Beth Israel Deaconess, Harvard Medical School; Michael Cocci, Beth Israel Deaconess, Harvard Medical School; Ari Moskowitz, Beth Israel Deaconess, Harvard Medical School; Benjamin S. Abella, Perelman School of Medicine at the University of Pennsylvania; David F. Gaieski, Sidney Kimmel Medical College at Thomas Jefferson University; Clifton W. Callaway, University of Pittsburgh; Jonathan Elmer, University of Pittsburgh; Jon C. Rittenberger, University of Pittsburgh; Kelly N. Sawyer, University of Pittsburgh; Patrick J. Coppler, University of Pittsburgh; Mary Ann Peberdy, Virginia Commonwealth University; Joseph Ornato, Virginia Commonwealth University; Joshua C. Reynolds, Michigan 
State University, College of Human Medicine; Henry Wang, University of Alabama at Birmingham; Michael C. Kurz, University of Alabama at Birmingham.

\section{REFERENCES}

1. Berdowski J, Berg RA, Tijssen JG, Koster RW. Global incidences of out-of-hospital cardiac arrest and survival rates: systematic review of 67 prospective studies. Resuscitation 2010;81: 1479-87.

2. Sasson C, Rogers MA, Dahl J, Kellermann AL. Predictors of survival from out-of-hospital cardiac arrest: a systematic review and meta-analysis. Circ Cardiovasc Qual Outcomes 2010;3: 63-81.

3. Sara JD, Eleid MF, Gulati R, Holmes DR Jr. Sudden cardiac death from the perspective of coronary artery disease. Mayo Clin Proc 2014;89:1685-98.

4. Lorusso $R$, Centofanti $P$, Gelsomino $S$, et al. Venoarterial extracorporeal membrane oxygenation for acute fulminant myocarditis in adult patients: a 5-year multi-institutional experience. Ann Thorac Surg 2016;101:919-26.

5. Ahn JM, Lee KH, Yoo SY, et al. Prognosis of variant angina manifesting as aborted sudden cardiac death. J Am Coll Cardiol 2016;68:137-45.

6. Cho YH, Kim WS, Sung K, et al. Management of cardiac arrest caused by acute massive pulmonary thromboembolism: importance of percutaneous cardiopulmonary support. ASAIO J 2014;60:280-3.

7. Chen YS, Lin JW, Yu HY, et al. Cardiopulmonary resuscitation with assisted extracorporeal life-support versus conventional cardiopulmonary resuscitation in adults with in-hospital cardiac arrest: an observational study and propensity analysis. Lancet 2008;372:554-61.

8. Lasa JJ, Rogers RS, Localio R, et al. Extracorporeal cardiopulmonary resuscitation (E-CPR) during pediatric in-hospital cardiopulmonary arrest is associated with improved survival to discharge: a report from the American Heart Association's Get With The Guidelines-Resuscitation (GWTG-R) registry. Circulation 2016;133:165-76.

9. Younger JG, Schreiner RJ, Swaniker F, Hirschl RB, Chapman RA, Bartlett RH. Extracorporeal resuscitation of cardiac arrest. Acad Emerg Med 1999;6:700-7.

10. Morimura N, Sakamoto T, Nagao K, et al. Extracorporeal cardiopulmonary resuscitation for out-of-hospital cardiac arrest: a review of the Japanese literature. Resuscitation 2011;82:10-4.

11. Kim SJ, Kim HJ, Lee HY, Ahn HS, Lee SW. Comparing extracorporeal cardiopulmonary resuscitation with conventional car- diopulmonary resuscitation: a meta-analysis. Resuscitation 2016;103:106-16.

12. Stub $D$, Bernard $S$, Pellegrino $V$, et al. Refractory cardiac arrest treated with mechanical CPR, hypothermia, ECMO and early reperfusion (the CHEER trial). Resuscitation 2015;86:88-94.

13. Sakamoto T, Morimura N, Nagao K, et al. Extracorporeal cardiopulmonary resuscitation versus conventional cardiopulmonary resuscitation in adults with out-of-hospital cardiac arrest: a prospective observational study. Resuscitation 2014; 85:762-8.

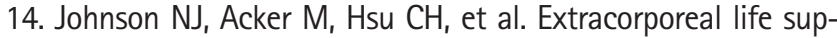
port as rescue strategy for out-of-hospital and emergency department cardiac arrest. Resuscitation 2014;85:1527-32.

15. Bellezzo JM, Shinar Z, Davis DP, et al. Emergency physicianinitiated extracorporeal cardiopulmonary resuscitation. Resuscitation 2012;83:966-70.

16. Lamhaut $L$, Jouffroy $R$, Soldan $M$, et al. Safety and feasibility of prehospital extra corporeal life support implementation by non-surgeons for out-of-hospital refractory cardiac arrest. Resuscitation 2013;84:1525-9.

17. Link MS, Berkow LC, Kudenchuk PJ, et al. Part 7: adult advanced cardiovascular life support: 2015 American Heart Association guidelines update for cardiopulmonary resuscitation and emergency cardiovascular care. Circulation 2015;132:S444-64.

18. Ortega-Deballon I, Hornby L, Shemie SD, Bhanji F, Guadagno E. Extracorporeal resuscitation for refractory out-of-hospital cardiac arrest in adults: a systematic review of international practices and outcomes. Resuscitation 2016;101:12-20.

19. Tonna JE, Johnson NJ, Greenwood J, et al. Practice characteristics of emergency department extracorporeal cardiopulmonary resuscitation (eCPR) programs in the United States: the current state of the art of emergency department extracorporeal membrane oxygenation (ED ECMO). Resuscitation 2016; 107:38-46.

20. Coppler PJ, Sawyer KN, Youn CS, et al. Variability of post-cardiac arrest care practices among cardiac arrest centers: United States and South Korean dual network survey of emergency physician research principal investigators. Ther Hypothermia Temp Manag 2017;7:30-5.

21. Kim YM, Youn CS, Kim SH, et al. Adverse events associated with poor neurological outcome during targeted temperature management and advanced critical care after out-of-hospital cardiac arrest. Crit Care 2015;19:283.

22. Donnino MW, Andersen LW, Giberson T, et al. Initial lactate and lactate change in post-cardiac arrest: a multicenter validation study. Crit Care Med 2014;42:1804-11.

23. Donnino MW, Rittenberger JC, Gaieski D, et al. The develop- 
ment and implementation of cardiac arrest centers. Resuscitation $2011 ; 82: 974-8$.

24. Recommended guidelines for uniform reporting of data from out-of-hospital cardiac arrest: the 'Utstein style.' Prepared by a Task Force of Representatives from the European Resuscitation Council, American Heart Association, Heart and Stroke Foundation of Canada, Australian Resuscitation Council. Resuscitation 1991;22:1-26. 